\title{
Drug Delivery Systems Based on Polymeric Micelles and Ultrasound: A Review
}

\author{
Rafeeq Tanbour ${ }^{1 \S}$, Ana M. Martins ${ }^{1 \S}$, William G. Pitt ${ }^{2}$ and Ghaleb A. Husseini ${ }^{1 *}$
}

\author{
${ }^{1}$ Department of Chemical Engineering, American University of Sharjah, P.O. Box 26666, Sharjah, United Arab \\ Emirates; ${ }^{2}$ Chemical Engineering Department, Brigham Young University, Provo, UT, USA
}

\begin{abstract}
Background: Anti-neoplastic drugs used for cancer treatment often produce damage to healthy cells, leading to severe side effects in patients undergoing chemotherapy. The encapsulation of these agents in nanoparticles reduces the adverse side effects of conventional chemotherapy on healthy tissues. Such nanoparticles, considered as drug delivery vehicles, are diverse and include micelles, liposomes, dendrimers, nanocapsules, nanospheres and others. Polymeric micelles have been widely researched as nanocarriers for hydrophobic drugs. They can be designed to have increased stability and blood circulation time, as well as binding specificity to certain receptors overexpressed on the surface of cancer cells. Once these drug-encapsulating nanoparticles reach the tumor site, an external stimulus, such as ultrasound, can be used to spatially and temporally trigger drug release. Methods: This review paper focuses on the recent advances of cancer drug delivery systems employing polymeric micelles and ul-
\end{abstract}

trasound. An extensive literature review was performed mainly using PubMed. The introduction explains how nanocarriers are related to chemotherapy and the several modalities of use for this application. Afterwards, the review focuses on polymeric micelles used for drug delivery, their advantages and disadvantages. Subsequently, the physics of ultrasound is briefly reviewed, as well as the way it interacts with polymeric micelles to trigger the delivery of the drug transported by these nanocarriers. The following section focuses on targeting, discussing the several ways by which the nanoparticles can be directed to the target cells, and there deliver their cargo. Finally, a selection of relevant in vitro and in vivo studies, as well as clinical trials, is presented and discussed. Conclusion: Although there are still several research studies to be performed before the combination of micelles and ultrasound can enter clinical trials, the future of controlled delivery using this drug delivery system is promising as a way to reduce the mortality and morbidity of cancer and the noxious side effects of conventional chemotherapy.

Keywords: Cancer, chemotherapy, drug release, polymeric micelles, triggered release, ultrasound.

\section{INTRODUCTION}

In the human body, cells are continuously regenerated in a cycle where older cells die in accordance with genetic programming that defines their life cycle. In some cases mutations occur in genes related to regulation, cell division and death, which may lead to abnormal or uncontrolled cell growth, thus producing tumors in tissues and organs $[1,2]$. For example, one of the main players in the cell cycle regulation is the gene $\mathrm{p} 53$, and it has been observed that mutations in this gene lead to $\mathrm{p} 53$ proteins that acquire oncogenic properties. The mutant proteins participate in processes that promote metastasis spreading of abnormal cells, proliferation and cell survival, which are characteristics of malignant tumors, i.e., cancers [3].

Cancer is the leading cause of death in Europe and North America, while being the second leading cause of death in the less developed countries, especially in Africa $[4,5]$. The treatment of cancer often involves subjecting the patient to a chemical treatment (chemotherapy), which usually requires repeated doses of drugs. Although chemotherapy may be effective in cancer treatment, it also has unwanted effects on healthy cells and organs [6]. Hence, scientists have been searching for better drug delivery methods in order to decrease these side effects, without compromising the efficacy of the chemotherapeutic drug in killing cancer cells. Such drug delivery systems (DDS) include the use of nanoparticles that sequester the drug inside their structure and release it at the tumor site using internal and/or external stimulus. This helps in decreasing the damage caused to healthy cells because it localizes the effect of chemotherapy on the tumor cells by controlling the time and space in which the drugs are released [7-10].

*Address correspondence to this author at the Department of Chemical Engineering, American University of Sharjah, PO Box 26666, Sharjah, United Arab Emirates; Tel: +971-6-515-2970; Fax: +971-6-515-2979; E-mail: ghusseini@aus.edu

${ }^{\S}$ These authors contributed equally to this review paper.

$1381-6128 / 16 \$ 58.00+.00$
There are many types of nanosized drug carriers that can be used in chemotherapy, including micelles, liposomes, nanospheres, nanocapsules and dendrimers (Fig. 1). Following liposomes, micelles are the second most commonly used nanoparticles in drug delivery [11], and are the focus of this review. Controlled drug delivery via liposomes has been recently reviewed by our group, particularly the combination of these lipid bilayer-based nanocarriers and the use of ultrasound (US) as a triggering mechanism [12, 13]. Drugs can also be delivered from solid or solid-shell particles, such as nanospheres or nanocapsules, made of a polymeric shell or matrix within which the drug is encapsulated. Dendrimers are highly branched molecules, which form cavities where the drug can be sequestered. The drug can also be conjugated to the exterior surface of the dendrimer [14].

The present review is concerned with drug delivery from micelles. Micelles are colloidal dispersions of self-assembled amphiphilic molecules, i.e., molecules with two distinct regions with opposite affinities towards a solvent, e.g. hydrophilic and hydrophobic [15]. The sizes of these assemblies are determined by their composition and associated packing geometry, and usually range from 5 to 100 $\mathrm{nm}$ in diameter [15]. Polymeric micelles are made up of polymers that impart specific characteristics. Block copolymers of amphiphilic composition self-assemble into core-shell structures with the hydrophilic blocks forming the external layer. The type of drugs that can be encapsulated inside these carriers depends on the micelle geometry and the hydrophobic character of the drugs that partition to the core [16].

Modifications on drug carrier systems can improve the results of the drug treatment. The modifications can be done to the carrier itself, for example by adding targeting moieties to improve their capability to bind to tumor cells. This is called active or ligand targeting, and one example is the folate moiety, which binds the folate receptor overexpressed on several types of cancer cells. Additionally, external factors including ultrasound (US), hyperthermia

\section{(C) 2016 Bentham Science Publishers}

"The published manuscript is available at EurekaSelect via http:// www.eurekaselect.com/openurl/content.php? genre $=$ article $\&$ doi $=[10.2174 / 1381612822666160217125215] . "$ 

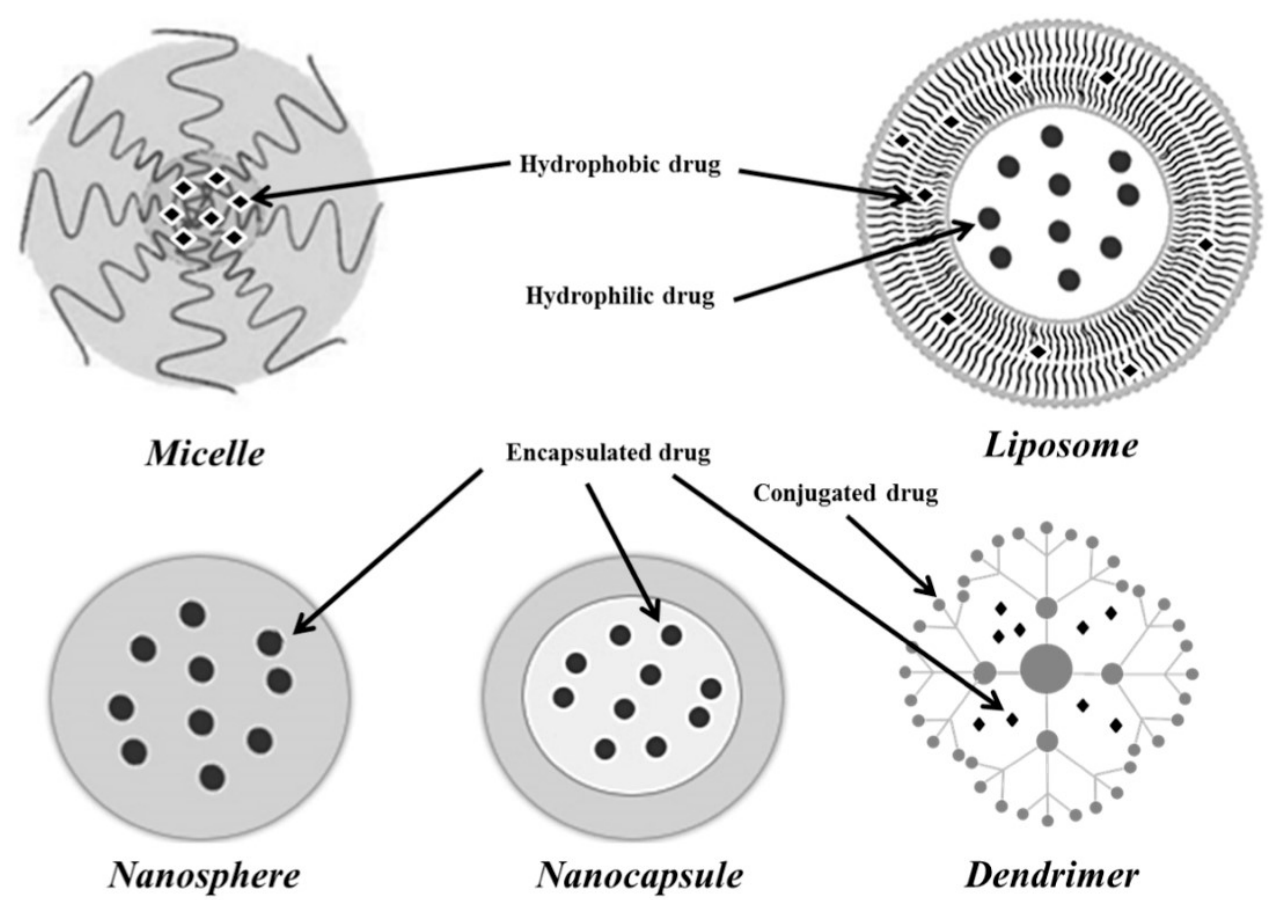

Nanocapsule

Dendrimer

Fig. (1). Different types of nanoparticles used as drug carriers [17].

and magnetic fields, can be used to trigger the drug delivery, a process called triggered targeting [17, 18].

Some of the most common drugs used in chemotherapy belong to the anthracycline family, and Doxorubicin (Dox) is widely used with high efficacy against several types of cancer [19]. However, it has several noxious side effects including cardiotoxicity and nonspecific action throughout the body $[20,21]$.

\section{POLYMERIC MICELLES}

Micelles, especially polymeric micelles, have been extensively studied as drug carriers nanosystems [6]. The classification of micelles is based on the type of intermolecular forces involved in their formation. In general, there are three types of micelles: amphiphilic micelles with predominance of hydrophobic interactions, polyion complex micelles which mainly have electrostatic interactions, and micelles stemming from metal complexation [22]. Polymeric micelles belong to the first category, and as mentioned, consist of amphiphilic block copolymers that selfassemble into core-shell structures.

The term micelle was first used to describe the self-assembled structures of small surfactant molecules [23]. At low concentrations, these molecules exist as monomers but when concentration increases, their hydrophobic tails tend to avoid water, thus aggregating in structures in which the hydrophobic parts are shielded, while the hydrophilic ones are exposed to water. This process, which is thermodynamically and entropically favorable, is called micellization [24]. The concentration at which micelles are formed is called critical micellar concentration (CMC) [25]. The micelle stability is driven by van der Waals forces between the hydrophobic regions [15], and the establishment of hydrogen bonds between the hydrophilic shell and water molecules [26]. Hence, micelles are characterized by a core, resulting from the aggregation of the hydrophobic regions, and a shell composed of the hydrophilic parts, exposed to the aqueous medium. The core of the micelle can solubilize poorly soluble drugs, while polar molecules can be adsorbed on the surface [9].

Polymeric micelles are, similar to low-molecular-weight surfactantbased micelles, characterized by a hydrophobic core - hydrophilic shell/corona structure. These micelles are made of amphiphilic block copolymers, developed as a result of the advances in polymer chemistry that occurred during the last 3 decades of the $20^{\text {th }}$ century. These copolymers allow the formation of a wide variety of nanostructures, and have several applications not only in pharmaceutical sciences, but also in coatings and paint formulations and personal care products, among others [27]. Amphiphilic block copolymers consist of at least two regions with different chemical properties, which allow them to self-assemble into micelles that may serve as nanocarriers to poor water-soluble compounds [28].

By the end of the 1990s, most micellar pharmaceutical research had focused on A-B diblock copolymers. Triblock copolymers with a structure A-B-A, have also been extensively studied in the last few decades, since they also self-assemble into micelles which can be used as nanocarriers [26, 29, 30]. The most used hydrophilic blocks are poly(ethylene glycol) (PEG) and poly(ethylene oxide) (PEO), while the core-forming hydrophobic blocks may be poly(Lamino acids) (e.g., poly(L-aspartate), poly(L-lysine) and derivatives, poly(esters) (e.g., poly(lactic acid), poly(-caprolactone), poly(glycolic acid) or poly(propylene oxide) [26, 28, 31].

Within the family of polymeric micelles, one of the most widely studied, for drug delivery purposes, is the family of Pluronic ${ }^{\circledR}$-based micelles, which is available commercially [28, 29, 32]. These are triblock copolymers composed of poly(ethylene oxide) (PEO) and poly(propylene oxide) (PPO), PEO-PPO-PEO, which when dissolved in water form a hydrophobic core and a hydrophilic shell, as illustrated in Fig. (2). Hence, hydrophobic drugs can be encapsulated inside the core of the micelle $[6,33]$. An advantage of using Pluronic ${ }^{\circledR}$ micelles as chemotherapy carriers (compared to other carriers) is their inherent size (10 to $200 \mathrm{~nm}$ ), which allows them to escape renal excretion and yet still extravasate at the tumor site. Additionally, the incorporation of the drugs inside their core is a simple process due to the hydrophobichydrophobic interactions between the drug and the PPO core [29, 34]. Furthermore, micelles are easy to prepare, their shelf life is long [29] and, at low concentration, they can sensitize multi-drug resistance (MDR) in cancer cells [35-37]. One of their main disadvantages, however, is that they disassociate if diluted below their 


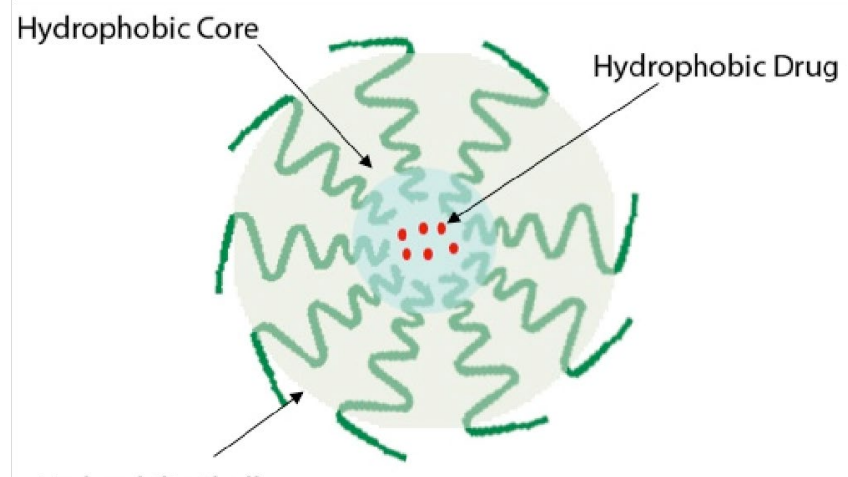

Hydrophilic Shell

Fig. (2). The structure of a polymeric micelle [33]. The hydrophobic polymer forms the core of the micelle, where the drug is encapsulated, while the hydrophilic chains face the outside, providing stability.

CMC, which makes them unstable when diluted to low concentrations, such as when injected into blood [34, 38].

The literature suggests the importance of determining the micellar stability in order to know if their structure can release and reencapsulate the drugs once the micelles reach the intended site. Also, it is important to study the effect of stability in relation to the rate of elimination of Pluronic ${ }^{\mathbb{B}}$ chains from the body after the drug has been released at the tumor site. The stability of Pluronic ${ }^{\mathbb{R}}$ micelles can be enhanced by cross-linking their core via emulsion polymerization using poly(N,N-diethyl acrylamide) (pNNDEA) as an example $[38,39]$. The stability of these micellar structures can be determined using the fluorescent probe diphenyl-1,3,5hexatriene (DPH) and dynamic light scattering [38, 40].

This review focuses on Pluronic ${ }^{\circledR}$ P105 which is the most common copolymer used in acoustically activated micellar drug delivery. These micellar carriers are ideal for US-triggered drug release because they are sensitive to low-frequency US, releasing the drug, and the drug can be quickly re-encapsulated when the insonation stops [30]. However, other polymeric micelles have been studied as US-sensitive nanoparticles, including the diblock copolymers poly(ethylene oxide)-block-poly(2-tetrahydropyranyl methacrylate) (PEO-b-PTHPMA), poly(ethylene oxide)-blockpoly[1(isobutoxy)ethyl methacrylate] (PEO- $b$-PIBMA), poly (ethylene oxide)-block-poly[(2-tetrahydrofuranyloxy)ethyl methacry-late] (PEO-b-PTHFEMA), poly(ethylene oxide-blockpoly(methyl methacrylate) (PEO- $b$-PMMA) [41], poly(lactic acid)blockpoly(ethylene glycol) (PLA-b-PEG) [42] and others which are further discussed in Section 5.1.

In Pluronic ${ }^{\circledR}$ P105, both flanking PEO blocks contain 37 repeat units and the central block has 56 units of PPO, i.e., the structure is $\mathrm{PEO}_{37-}$ $\mathrm{PPO}_{56}-\mathrm{PEO}_{37}$. The features that make Pluronic ${ }^{\circledR} \mathrm{P} 105$ a good choice over the other copolymeric micelles are its low toxicity, quick formation once dissolved in water, and stability above the CMC due to its hydrophobic core [6]. In this case, the CMC is defined as the minimum concentration of block copolymer that will result in micelle formation. The stability of these micelles is a function of the $\mathrm{CMC}$, especially when the copolymer is diluted in bodily fluids. At room temperature, the $\mathrm{CMC}$ of Pluronic ${ }^{\mathbb{B}} \mathrm{P} 105$ is close to $1 \mathrm{wt} \%$ [34]. The method of incorporating anti-neoplastic chemotherapeutics inside P105 micelles is relatively easy. First, a filtered stock solution of P105 of known concentration is prepared in a phosphate buffered saline (PBS) solution. Subsequently, a solution of the drug is added to the micellar solution, which results in micelles encapsulating the drug at a prescribed concentration [43].

\section{ULTRASOUND}

Ultrasonics is a branch of acoustics that studies sound pressure waves in a range of frequencies higher than the upper limit of human hearing, which is considered to be $20 \mathrm{kHz}$ [44].

Ultrasonic waves have the same physical properties as other waves, so they can be reflected, absorbed, refracted and focused [44].

Ultrasound has been widely used in medicine mainly as an imaging technique [45], but its use in the last three decades has dramatically increased due to the discovery of possible therapeutic applications, especially in cancer treatment. It has been observed that US has a synergistic effect on chemotherapeutic efficiency, while contributing to a decrease in the side effects of conventional chemotherapy [29]. Ultrasound can be categorized as high-intensity or low-intensity, and high-frequency $(>1 \mathrm{MHz})$ or low-frequency $(20-100 \mathrm{kHz})$. Lowintensity US is used for medical imaging and, to a lesser extent, for therapeutic purposes, while high intensity US is studied as a treatment directed toward many types of cancers. For a given intensity, higher frequency produces better imaging resolution, but the penetration depth of the wave decreases [46]. Recently, high frequency US (HFUS) is gaining more attention in cancer treatment due to the fact that it is easily focused on the tumor in comparison with low frequency US (LFUS) [47-49]. This characteristic of HFUS aids in efficiently controlling the release of chemotherapeutic agents from drug carriers, as will be explained shortly. Furthermore, as already stated, an increase in the US frequency allows the acquisition of clearer images of the tumor, which helps in directing the drug release process without the need for additional instrumentation [50]. Additionally, HFUS enhances the drug uptake in cells and tissues and facilitates the dissolution of blood clots for the treatment of strokes [51]. Another important US advantage is the non-invasive nature of this technique which avoids the disadvantages and complications of surgery [45].

When US is used in DDS it may cause both thermal and nonthermal effects on the body organs and tissues. The thermal effect is usually referred to as hyperthermia and is a result of the energy absorption by tissues and body fluids, causing a rise in temperature that may lead to the death of healthy cells. By selecting the proper US parameters, including its frequency and intensity, hyperthermia can be controlled and used as an effective modality in cancer treatment $[6,12,52]$. Furthermore, in some DDS, the drug carriers are synthesized to exhibit sensitivity to changes in temperature, such as temperature sensitive liposomes. Using US to heat the tumor site, temperature-sensitive drug carriers found in the vicinity of the tumor will be induced to release their content, thus mediating the process of drug delivery [53-55].

The non-thermal effects of US are mostly related to the cavitation of gas bubbles, which is their oscillation and possible collapse in an acoustic field [12, 52]. Several studies attribute the release of molecules from micelles exposed to US to ultrasonic cavitation [6]. 
There are two main categories of cavitation: stable cavitation which is the continuous oscillation of the bubbles without collapsing, and collapse (or inertial) cavitation, where the bubbles collapse aggressively, generating shock waves and causing large local temperature and pressure increases. Stable cavitation occurs at low US intensities, while collapse cavitation happens at higher intensities $[56,57]$. When a microbubble collapses, a shock wave is generated along with an extreme increase in the local temperature for a very short period. This shock wave propagates in the surrounding medium, where micelles may be found. If micelles happen to be in the vicinity of the shockwave, they get destroyed as the polymeric chains, forming the micellar structure, lose their association due to the high energy generated by the wave. This leads to the release of the encapsulated drug [58]. The effect of a collapse cavitation shock wave on a micelle is illustrated in Fig. (3).

The US-triggered release of drugs from micelles can be divided into three phases. The first phase is a rapid release of most of the encapsulated drug. The second phase entails a slower release, while the third and final phase includes a reformation of the micelle and reencapsulation of some of the drug molecules. The graphic in Fig.

(4) shows these three phases [59].

A very promising aspect of the use of US as a trigger mechanism is that the drug is quickly re-encapsulated into reformed micelles when the US is turned off [39]. The fraction of drug that does not enter nearby cancer cells can re-enter the micelles, hence decreasing its effect on healthy cells in other locations in the body.

\section{TARGETING}

In micellar systems there are many factors that can be studied and manipulated to achieve the best delivery, including micellar composition, tumor location and the drug itself. Micellar nanocarriers may be used in passive, triggered and ligand (also referred to as active) targeting. The mechanisms involved in each general category should be understood to obtain and leverage the best treatment results when using nanoparticles in chemotherapy $[6,60]$.

In passive targeting the particles circulating in the bloodstream accumulate in a desired location based on mechanical flow and deposition, not on chemistry. Passive targeting to tumors is based on the increased permeability of the vasculature observed in some tumors. This permeability varies depending on the condition and the type of tissue and tumor. The presence of a leaky vasculature at the site of the tumor facilitates the extravasation of the nanoparticles carrying the chemotherapeutic agent, which will enhance the relative release of drugs to the region and increase the local concentration, resulting in more cellular uptake. This phenomenon of particle collection is known as the enhanced permeation and retention (EPR) effect [61, 62].

In addition, tumor-targeting may be enhanced by modifying the micelle surface with ligands that are specific to receptors present on the membrane of the cancer cells [63]. This type of modification, called ligand targeting, is an active targeting strategy that can be generally defined as the use of targeting moieties or ligands to attach nanoparticles to the target site [64]. Choosing the best targeting moiety is important because it affects nanoparticle properties including circulation half-life, cellular uptake, binding affinity, and extravasation [65]. Several types of moieties have been investigated including small peptides, antibodies (immunomicelles), hormones, aptamers and low molecular weight ligands such as folate [66]. Folate (folic acid, vitamin B9), a molecule essential for several processes including cell growth and division, is one of the most studied small molecules used in ligand targeting [67-70]. Its use in drug delivery and targeting is a result of its strong binding to the folate receptor (FR), which is widely expressed on the surface of some cancer cells, including ovarian, brain, kidney, breast, lung and others $[71,72]$. Folate binds with high affinity to the FR. Employing folate in a DDS renders it more useful for both imaging [73] and therapeutic purposes [74].

Another approach to active tumor targeting is triggered targeting, by which the drug release from micelles to the intended tumor is enhanced by stimuli, including electric field, $\mathrm{pH}$, temperature and ultrasound [75]. The selection of the trigger to be used depends on the type of micelles, the anti-neoplastic agent, and the surrounding environment of the tumor [25]. Ultrasound, which has been previously discussed, is considered an attractive trigger mechanism in drug delivery because it is non-invasive and capable of disrupting micelles and releasing the drug from their core [76].
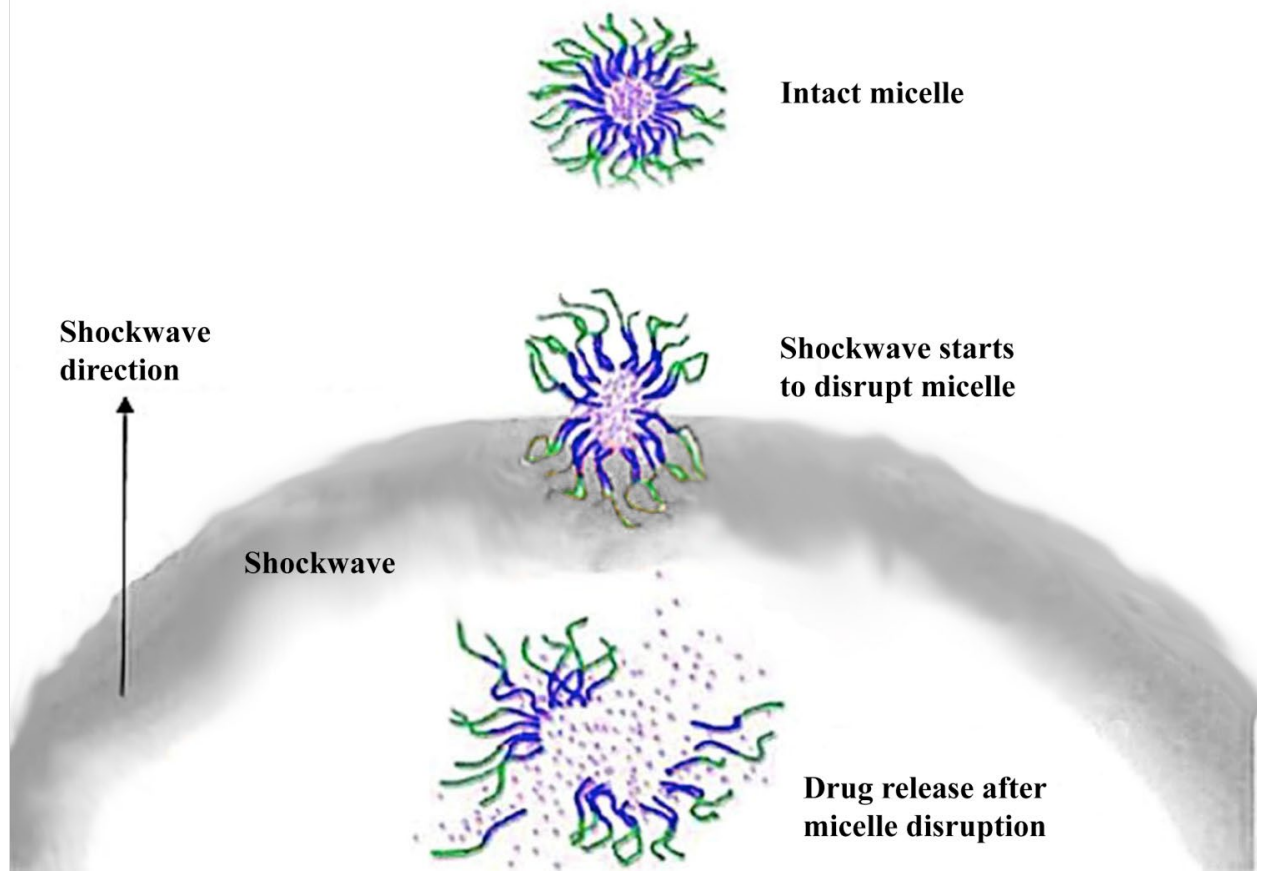

Fig. (3). The effect of cavitation events on the release of drugs from polymeric micelles [29]. 


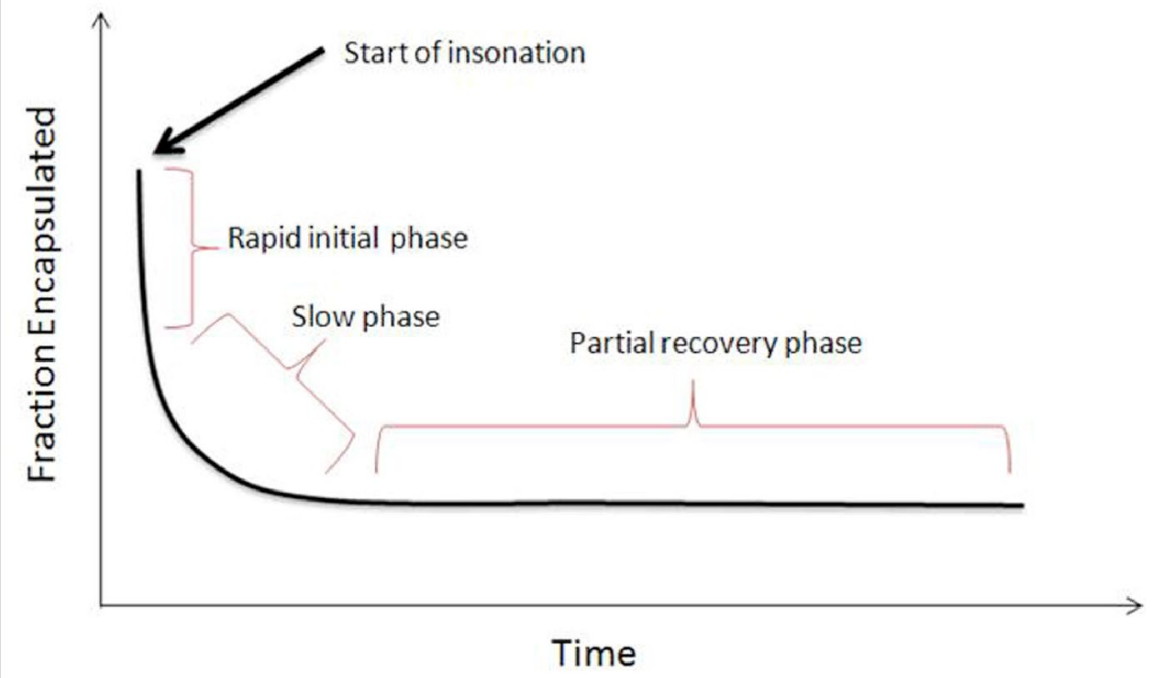

Fig. (4). Phases of drug release from micelles in the presence of US [56]. The graph shows the effect of cavitation events on the release of drugs from a collection of polymeric micelles in a large volume [29].

\section{RELEVANT IN VITRO AND IN VIVO WORK AND CLINICAL STUDIES}

There are several scientific reports describing the use of ultrasound to release drugs from polymeric micelles in vitro and in vivo $[6,12$, $29,30,76]$. This section will focus on some of the most important studies in this area.

\subsection{In Vitro Studies}

Several in vitro studies have been published regarding the release of drugs from micelles upon the application of US, and different factors and environments were studied to improve this DDS. Some of these factors are related to US, including power density, frequency and duration of sonication, while others are related to the polymer properties and the drugs used. All of these studies aim to make these systems suitable and safe for human use [12, 30]. Additionally, in vitro studies using cell cultures are very useful in order to design in vivo experiments.

Many in vitro drug release and cancer treatment research studies use fluorescence techniques to quantitate drug release in one of two ways: (i) the offline method where the sonication of the sample is done externally and then the fluorescence level is measured afterwards; and (ii) the online method which involves the use of a sonication chamber fitted with a fluorescence measurement device [57]. In the sonication chamber, the ultrasonic exposure of the sample happens while simultaneously and continuously monitoring the fluorescence level using some kind of fluorescence detector. Doxorubicin is an example of a fluorescent chemotherapeutic agent, absorbing light at $488 \mathrm{~nm}$ and emitting fluorescent light between 530 and $630 \mathrm{~nm}[77,78]$. Ultrasound is applied to drug carriers using a transducer with a known frequency, applying a power density measured by a hydrophone. Fiber optics guide laser excitation light to the sample, and also collect the fluorescent emissions during the application of US; the data are usually collected, stored and analyzed offline $[79,80]$. A simplified diagram of such an US chamber with fluorescence detection is shown in Fig. (5).

\subsubsection{Factors Affecting Drug Release}

The effect of US frequency on the release of drugs from polymeric micelles has been widely studied by Pitt and co-workers (reviewed in $[6,12,30])$, and the results show that the release of drugs encapsulated in polymeric micelles increases as the ultrasonic frequency decreases (at constant intensity). Husseini et al. [76] prepared Pluronic ${ }^{\circledR}$ P105 micelles containing either Dox or its paramagnetic analogue Ruboxyl, and exposed them to different frequencies of US, in the range of 20 to $90 \mathrm{kHz}$. The group also studied the effect of varying the power density $\left(0\right.$ to $\left.3 \mathrm{~W} / \mathrm{cm}^{2}\right)$ and observed that, at a constant frequency, an increase in power density caused an increase in drug release. It was also observed that at micellar concentrations lower than $0.1 \mathrm{wt} \%$, the release was higher compared to the higher Pluronic ${ }^{\circledR}$ concentrations. In studies of varying pulse duration, it was observed that, above a threshold of 0.1 $\mathrm{s}$, there was increased release with increasing pulse duration up to $0.5 \mathrm{~s}$. Above this pulse length, the release was not different than with application of continuous wave (CW) US. Furthermore, it was observed that the drug was re-encapsulated between pulses, which suggests that perhaps in vivo, released drug that is not taken up by cells may re-enter the micelle and decrease the harmful effects associated with Dox on adjacent non-cancerous tissues. A later study [81] using a fluorescence detection exposure chamber similar to the one described earlier (Fig. 5) investigated the release and reencapsulation of Dox from Pluronic ${ }^{\circledR} \mathrm{P} 105$ micelles upon exposure to $20-\mathrm{kHz}$ US at a power density of $58 \mathrm{~mW} / \mathrm{cm}^{2}$. The results showed that exposure to US for a period shorter than $0.1 \mathrm{~s}$, did not induce release of Dox from the micelles, similar to the result obtained in the previous study [76]. Above this threshold, the release increased with the pulse length, up to $0.6 \mathrm{~s}$. On the other hand, the minimum off pulse for the re-encapsulation to occur was $0.1 \mathrm{~s}$. The data were used to develop a mathematical model for this polymeric system and the model showed a zero-order release and first-order re-encapsulation kinetics. The model was further investigated by the same group [59] and four simultaneous mechanisms were proposed using the assumption of four different micelle sizes. These mechanisms were divided into two parts, the first included destroying and re-assembly of the micelle, while the second included the releasing and reencapsulation of Dox. It was assumed that the micelles' destruction was due to the cavitation events. The kinetic model built for these mechanisms was consistent with the assumption that the collapse cavitation plays a strong role in the release phenomena observed experimentally. Another modeling study by the same group [82] used an artificial neural network (ANN) model to predict Dox release from the same type of micelles, upon exposure to $20-\mathrm{kHz}$ US at different power densities. This model was then used to optimize the US parameters needed to achieve a target drug release at the tumor site. 


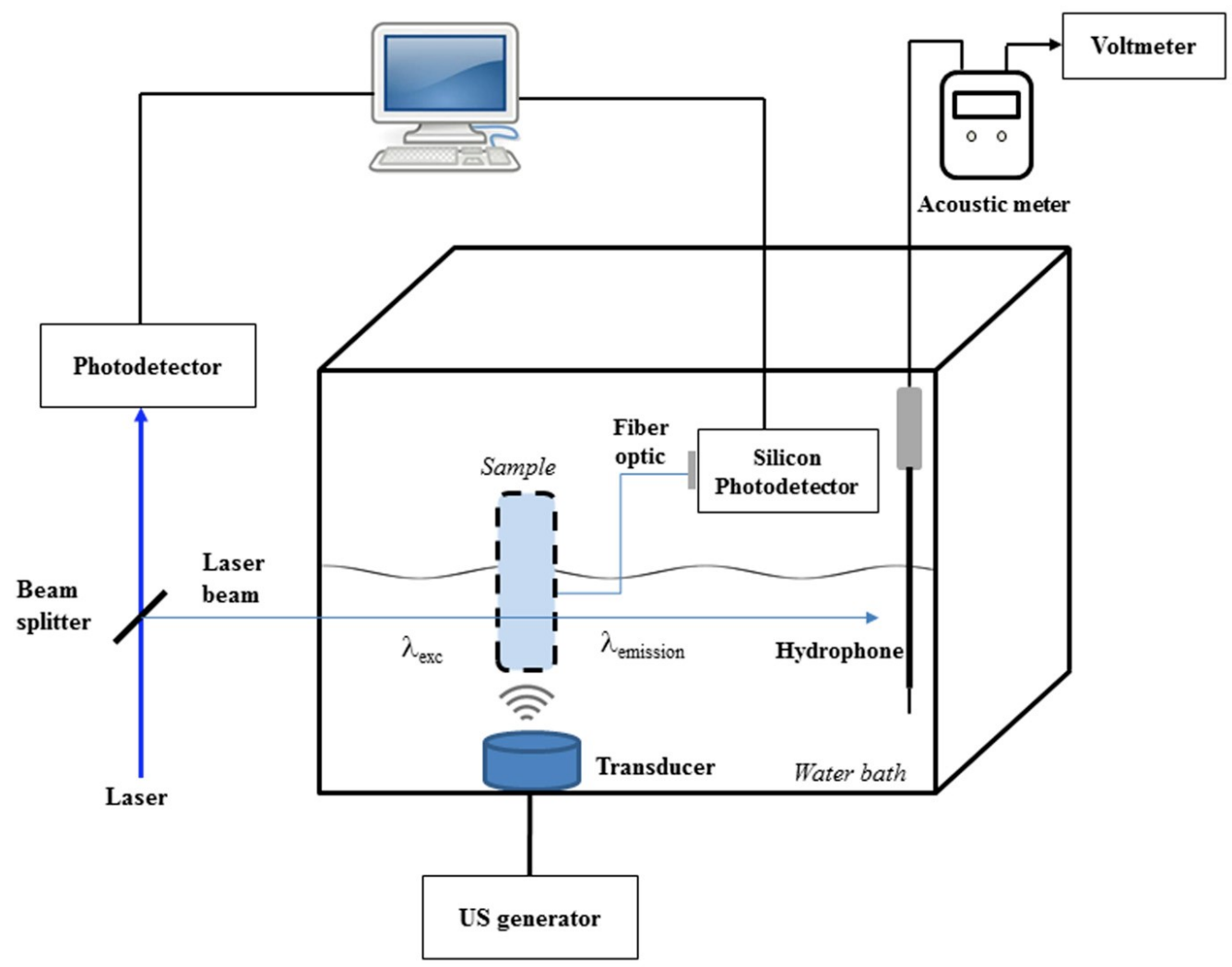

Fig. (5). Ultrasonic exposure chamber [76, 79]. The schematic shows an ultrasonic exposure chamber with fluorescent detection. The sample - micelles with an encapsulated fluorescent drug - is kept in a glass cuvette. The fluorescence of the drug is excited with a laser beam. Drug release is induced by an US probe situated under the cuvette, and is quantified by measuring the fluorescent emission detected by a fiber optic probe, filtered and digitized in a silicon photodetector. The laser power is monitored using a monitor photodetector. Additionally, a hydrophone is used to measure the characteristics of the ultrasound wave, such as frequency and amplitude, by converting it into electric dilution in blood led researchers to design and synthesize stabilized polymeric signals.

micelles. Pruitt et al. [38] synthesized NanoDeliv ${ }^{\mathrm{TM}}$, P105 micelles stabilized with the cross-linking agent pNNDEA, and the same group later $[39,83]$ compared the release from these stabilized micelles and the unstabilized Pluronic ${ }^{\circledR}$ P105. Using $70-\mathrm{kHz}$ US they observed that the release from both types of micelles was similar [39]. Another study using the same US frequency [83] compared the Dox release from unstabilized and stabilized polymeric micelles

(NanoDeliv ${ }^{\mathrm{TM}}$ and micelles of PEO- $b$-poly(N-isopropylacrylamide)$b$-poly(oligolactylmethacrylate) with stabilized cores). The study of the release kinetics showed that the Dox release rates from unstabilized micelles were significantly higher than those from stabilized ones, but that the onset of release occurred at the same ultrasonic power density for all micelles studied.

A different type of stabilized micelles was developed by Ugarenko et al. [84], who studied the release of Dox and formaldehydereleasing prodrugs from stabilized Pluronic ${ }^{\mathbb{B}}$-DSPE-PEG2000 mixed micelles, using $20-\mathrm{kHz}$ US at high power densities $\left(100 \mathrm{~W} / \mathrm{cm}^{2}\right)$. They observed that when micelles were formed, $60 \%$ of Dox was encapsulated with a retention half-life of approximately 12 hours. It was observed that at these high US power densities, $7-10 \%$ of the encapsulated Dox was released. On the other hand, the formaldehyde-releasing prodrugs were not encapsulated inside the micelles, but they could be used separately to enhance the formation of Dox-DNA adducts in tumor tissues.

Besides Pluronic ${ }^{\circledR}$ P105, other block copolymers have been used to form drug delivering micelles, and the effect of US on the release of workers [42] synthesized polymeric micelles using a block copolymer PLA- $b$-PEG, loaded with the Nile Red stain. They used high-intensity focused ultrasound (HIFU) to trigger the release from these nanoparticles. Their results showed that the HIFU can trigger the irreversible release of Nile Red through mechanisms related to transient cavitation. The release could be controlled by tuning the properties of US including intensity, time and the location where it is focused. Recently, Xuan and co-workers [41] studied the effect of $1.1-\mathrm{MHz}$ HIFU in several different polymeric micelle formulations, using PEO- $b$-PTHPMA, PEO- $b$-PIBMA, PEO- $b$-PTHFEMA, and PEO- $b$-PMMA block copolymer micelles. Their results showed that the PEO- $b$-PMMA micelles are more resistant to disruption induced by HIFU, when compared to the other formulations. The same group [85] described a DDS based on 1.1-MHz HIFU and on micelles of a diblock copolymer of PEO and poly(2-(2methoxyethoxy)ethyl methacrylate) ( $\left.\mathrm{PMEO}_{2} \mathrm{MA}\right)$ with some added units of the 2tetrahydropyranyl methacrylate (THPMA). The results showed an amplification of the effect of HIFU exposure on the disassembling of the micelles. The exposure to HIFU leads to the hydrolysis of the hydrophobic THPMA groups into hydrophilic methacrylic acid, thus increasing the lower critical solution temperature of the core $\mathrm{PMEO}_{2} \mathrm{MA}$ and leading to the disassembly of the micelles.

Wang et al. [86] also studied the effect of 1.1-MHz HIFU in the disruption of micelles of a diblock copolymer made of PEO and poly(2-tetrahydropyranyl methacrylate) (PEO- $b$-PTHPMA). The micelle disruption was due to the US-induced hydrolysis of the PTHPMA, which was attributed to the cleavage of tetrahydropyranyl groups. 
The HIFU-triggered release of hydrophobic molecules from noncross-linked and core cross-linked micelles of PEG- $b$-poly[N(2hydroxypropyl) methacrylamide-lactate] (PEG- $b$ p(HPMAmLac(n))) was recently reported [87]. The results showed that both continuous wave (CW) high frequency US and pulsed 1.5$\mathrm{MHz}$ HIFU triggered the release of the hydrophobic molecule Nile Red from both cross-linked and non-cross-linked micelles. However, the release from the stabilized, cross-linked micelles was lower than that observed from non-cross-linked ones. The results were not attributed to cavitation and/or hyperthermia as the main release mechanisms; instead the authors proposed that the radiation forceinduced shear forces at the walls of the exposure chamber causes the transient destabilization of the micelles, leading to release of their contents. The release from micelles with a densely crosslinked core is lower, since these micelles are stabilized and are more difficult to destabilize than non-stabilized micelles.

Targeted polymeric micelles combined with US have also been recently described as an efficient DDS [60]. Pluronic ${ }^{\circledR}$ P105 micelles conjugated to a folate moiety were synthesized and used to study the relationship between the intensity of $70-\mathrm{kHz}$ US and the kinetics of Dox release. The results suggested, similar to the case of non-targeted micelles, an important role of cavitation in the drug release. Additionally, it was observed that, using the same US conditions, the release from folated micelles was higher than from nonfolated ones [78]. The same author described a model to predict the acoustic drug release from non-targeted and folate-targeted polymeric micelles, using $70-\mathrm{kHz}$ US at several different power densities [88].

\subsubsection{Polymeric Micelles and US: Effect on Cell Cultures In Vitro}

The first in vitro study on the effects of the use of micelleencapsulated drugs on cancer cell cultures in conjunction with US, was described by Munshi et al. in 1997 [89], using leukemia HL-60 human cancer cells. The group reported a synergistic effect when using $80-\mathrm{kHz}$ US and Dox encapsulated in Pluronic ${ }^{\circledR} \mathrm{P} 105$, noting that the drug $\mathrm{IC}_{50}$ decreased in the presence of US.

The same cell line was used by Husseini et al. [43] to study the in vitro effect of Dox on the cellular DNA. As mentioned Dox is one of the most effective drugs against cancer, but it has severe side effects. This gave rise to the need for more directed DDS, hence the use of targeted micelles. Dox was delivered in two ways: the first was by directly adding it in its free state, and the second was by encapsulating it inside Pluronic $\mathbb{R}$ P105 micelles. The structure of the cellular DNA was measured by the comet assay, after experiments with and without exposure to $70-\mathrm{kHz}$ US. No DNA damage was observed in cells exposed to micellar-encapsulated Dox in the absence of US, but free drug produced some DNA damage. However, it was observed that when Dox was delivered encapsulated in micelles under exposure to US, the DNA damage was significantly higher and $96 \%$ of the cancer cells were dead after 2 hours of exposure. Importantly, this study also established that the mode of cell death was apoptosis and not necrosis.

Marin et al. [90] studied the effect of CW and pulsed 20-kHz US on the uptake by HL-60 cells of free and Pluronic ${ }^{\circledR}$ encapsulated Dox. The results showed that both free and encapsulated drug uptake increased with power intensity, from 1.4 to $33 \mathrm{~mW} / \mathrm{cm}^{2}$. Using pulsed US it was observed that the Pluronic ${ }^{\circledR}$ micelles uptake increased with pulse duration, for 0.1 to $2 \mathrm{~s}$ pulses, while there was no significant effect on the uptake of free Dox. For both free and encapsulated Dox, there was no significant effect of the duration of the inter-pulse interval. The authors suggested two mechanisms for the US-induced uptake from Pluronic ${ }^{\mathbb{B}}$ micelles: (i) US-induced Dox release from micelles with an increase of free Dox in the medium; (ii) US-induced permeability of the cell membrane, with increase in the uptake of encapsulated Dox.

In another investigation, the relation between drug release and high frequency (1-MHz) US was examined [91]. The tests were conducted on different types of cancer cell lines including HL-60, drug-sensitive ovarian carcinoma and breast cancer MCF-7 cells. The group hypothesized that when US was applied, cavitation events occurred that could be monitored by quantifying the associated free radicals. It was observed that there was formation of radicals with changing frequencies and power densities. The cavitation threshold intensity increased as the US frequency increased, as evidenced by the formation of free radicals at different frequencies. It was noticed that at higher frequencies, although transient cavitation almost ceased to exist, drug release was still observed, indicating that drug release from micelles was not tied to transient cavitation. It was also found that the rate of cellular uptake of Dox was higher even at short time exposures to 1-MHz HFUS.

Howard and co-workers [92] synthesized micelles of methylcapped poly(ethylene oxide)-co-poly-(L-lactide)-tocopherol containing encapsulated Paclitaxel. They sonicated the micelles at $1 \mathrm{MHz}$ US with $1.7 \mathrm{~W} / \mathrm{cm}^{2}$ power density to study the efficacy of this DDS in killing human breast adenocarcinoma MCF-7 cells. The results showed that drug release from these micelles could be efficiently induced by US and also emphasized the importance of using micelles to encapsulate drugs, which aids in reducing the side effects observed when the drug is introduced in its free form.

Ugarenko et al. [84] examined Dox uptake by the breast cancer cell line MDA-MB-231 using Dox and formaldehyde-releasing prodrugs encapsulated in stabilized Pluronic ${ }^{\circledR}$-DSPE-PEG2000 mixed micelles. The results showed that, in the absence of US, the uptake of the micellar system was reduced when compared to that of free Dox. On the contrary, upon application of $20-\mathrm{kHz}, 100 \mathrm{~W} / \mathrm{cm}^{2} \mathrm{US}$, the drug was released from the micelles and the cellular uptake was increased.

Wan et al. [93] used a micellar formulation of methoxyPEGblockpoly(D,L-lactide) (MePEG- $b$-PDLLA) to encapsulate paclitaxel and study the effect of 4-MHz HFUS at $32 \mathrm{~W} / \mathrm{cm}^{2}$ power intensity on the cellular accumulation in drug-sensitive and nonsensitive cell lines. There was an increase in drug uptake in both cell lines, induced by US, and globally the results suggested that US facilitates the uptake and retention of micelle-encapsulated drug. 5.1.3. Mechanism of Drug Release/ Cell Uptake

In vitro release studies suggest three different mechanisms by which US enhances the drug release from micelles and uptake by cells [12]. Although this subject has been extensively studied, different studies have shown different results, suggesting that different cells lines may respond differently.

The first proposed mechanism considers that the drug release induced by US occurs outside the cells, and is followed by the drug entering the cells by a normal diffusion process. Most research done in the area does not support this mechanism.

The second mechanism - sonoporation - states that the cell membrane is transiently perturbed by the application of US, allowing for the transport of released or encapsulated drugs into the cells. Several in vitro studies provided evidence that the use of US both releases the drug from the micelles and creates transient pores in the cell membranes through which the drug can enter into the cell cytosol. Tachibana et al. [94] and Yamashita et al. [95] conducted several experiments to study the effect of US on the permeability of cell membranes. In one of their earlier studies [96], HL-60 cancer cells were sonicated for $30 \mathrm{sec}$ with $255-\mathrm{kHz}$ continuous US, with a power density of $0.4 \mathrm{~W} / \mathrm{cm}^{2}$, in the presence of the fluorescent dye merocyanine 540. Using electron microscopy, the authors observed the formation of pores in the cell membranes that were thought to be 
related to cell death. They later concluded that the cell death was caused by both the drug and the US treatment [95]. To confirm their theory, the same group conducted another experiment using the cytosine arabinoside drug with HL-60 cells sonicated at $48-\mathrm{kHz}$ US with a power density of $0.3 \mathrm{~W} / \mathrm{cm}^{2}$ [94]. The results of this study showed that cell death increased upon sonication, when compared to a sonicated control sample that was not exposed to the drug. This suggested that cell membrane permeability increased when subjected to US, allowing for the diffusion of the drug through the transient membrane pores into their cytosol and leading to their death [94, 95]. The effect of US on cell membrane permeability was later studied by Schlicher et al. [97, 98]. This group used DU145 prostate cancer and primary human astrocyte (HA) cells. The used US conditions were: 20 pulses of $24-\mathrm{kHz}$ US, each pulse with a duration of $0.1 \mathrm{~s}$, a $10 \%$ duty cycle and different acoustic amplitude pressures $(0.36,0.54$ and $0.71 \mathrm{~atm}$ ), which are a measure of the US power [97]. The study concluded that cavitation was responsible for multiple forms of membrane wounding, which increased with acoustic pressure (i.e. ultrasonic power density). For the highest pressure used, nuclear ejection through the damaged membrane was observed, followed by cell lysis, which suggested that the size of the pores formed increased with the increase of cavitation events, which was proportional to the sonication intensity. Later, a study done by Zhou et al. [99] used the voltage clamp technique to measure the size of the pores formed by the application of $1.075-\mathrm{MHz}$ US $(0.2 \mathrm{~s}, 0.3 \mathrm{MPa})$ in Xenopus laevis oocytes and determined these pores to have a $110 \pm 40 \mathrm{~nm}$ radius.

A third proposed mechanism considers that endocytosis is the main cause of increased cell death due to the US-induced enhanced chemotherapeutic drug uptake. Several studies showed that there are no significant cell membrane deformations upon application of mild ultrasonication, which excluded sonoporation as the main mechanism of drug release and uptake $[100,101]$. Results obtained by Muniruzzaman and co-workers [102] suggested that the uptake of micelles by cells occurs via fluid-phase endocytosis. The aim of their study was to test the effect of the aggregation state on the intracellular uptake of Pluronic ${ }^{\circledR}$ P105 micelles by HL-60 cancer cells. It was observed that, below the $\mathrm{CMC}$, the cell uptake increased with the increasing concentration of micelles in the incubation medium, while above the CMC, the intracellular uptake was less efficient. This suggested that below the $\mathrm{CMC}$ the single Pluronic ${ }^{\circledR}$ chains enter the cell via diffusion through the cell membrane, while the micelles enter the cell via endocytosis. Similarly, the studies by Rapoport et al. [103] and Sheikov et al. [104] provided some evidence of endocytotic events.

In summary, the in vitro studies proved the synergism between micelle-encapsulated drugs and US. The application of US increases the release of drugs from the nanocarriers and simultaneously induces transient pores in the cell membranes, which can enhance the uptake of drug by the cells. The endocytosis hypothesis, however, cannot be discarded, and both mechanisms must be considered. A recent review by Lentacker et al. [105] states that the mechanism of drug uptake through pores depends on the US parameters and that the US intensity may be modulated to regulate the pore size. Low intensity US may induce the uptake of some drugs (or drug carriers) by endocytosis, while short high intensity US pulses has the capability to induce sonoporation with subsequent drug uptake directly into the cytosol.

\subsection{In Vivo Studies}

In vivo studies are extremely important as preclinical experiments, and the most common animal models used to conduct in vivo experiments are mice and rats inoculated with a cancer cell line. The lab animals are then treated with Dox or other chemotherapeutic drugs, encapsulated in micelles or in a free form, to compare the efficiency of the different DDS, with and without exposure to US.
One of the first in vivo studies with micelles and US used a colon carcinoma ( $\mathrm{DHD} / \mathrm{K} 12 / \mathrm{TRb}$ cells) in a rat model to investigate the effect of Dox concentration, US frequency, and power density, among other variables [106]. The tumors were grown in the hind legs of the rat by injection with the tumor cells. After the tumors reached a desired size, the rat was treated with Dox at different concentrations, with and without application of US at the tumor site. The Dox was encapsulated into stabilized Pluronic micelles (NanoDeliv) and was administered weekly for a period of 6 weeks. One of the tumors on a leg was exposed to 20 or $70-\mathrm{kHz}$ US, while the tumor on the other leg was not US-treated, corresponding to a negative control. In the group without any drug, the growth of the bilateral tumors was similar and increased exponentially over time. For the groups treated with Dox, it was observed that the higher concentrations of encapsulated Dox were lethal to the animals: 4 and $5.33 \mathrm{mg} / \mathrm{kg}$ were lethal within 6 weeks, while $8 \mathrm{mg} / \mathrm{kg}$ killed the animals within 2 weeks. The treatment with micelles encapsulating Dox concentrations of 1.33 and $2.67 \mathrm{mg} / \mathrm{kg}$ was not lethal to the animals. In these cases, it was observed that the tumors treated with $70-\mathrm{kHz}$ US, $2 \mathrm{~W} / \mathrm{cm}^{2}$ had, on average, a lower growth rate than the non-insonated ones.

The same rat cancer model was used by Staples et al. [107] to investigate the effects of Dox encapsulated in stabilized Pluronic ${ }^{\mathbb{B}}$ micelles (NanoDeliv) triggered with low $(20 \mathrm{kHz})$ and medium (476 $\mathrm{kHz}$ ) frequency US. The study compared the results of treating the rats using micelles, with and without US, and it was observed that the tumor growth rate decreased when compared with tumors treated with micelles only (no US). The study also reported that tumor volumes could be fitted to an exponential growth model for both the control and insonated tumors at $20 \mathrm{kHz}$ and $476 \mathrm{kHz}$. No differences in growth rates were observed for $20-\mathrm{kHz}$ and $476-\mathrm{kHz}$ US (with the same mechanical index, MI, and time-averaged power density). In a similar study [108], the results showed that exposure to 20 - or 476$\mathrm{kHz}$ US increased the drug concentration in the tumor, compared to non-insonated micelle-treated ones. However, $12 \mathrm{~h}$ after drug injection, no difference in the concentration of Dox was observed between insonated and non-insonated tumors. Additionally, the variation of US frequency did not show any effect on tumor growth: it was observed that frequencies of $20 \mathrm{kHz}$ and $476 \mathrm{kHz}$ (with the same MI and time-averaged power density) produced very similar results with respect to Dox concentration in the tumors and tumor growth rate.

A study by Rapoport and co-workers [109] investigated the advantage of using Dox encapsulated in Pluronic micelles and $1 \mathrm{MHz}$ HFUS to treat immuno-compromised athymic $n u / n u$ mice, inoculated intraperitoneally with ovarian carcinoma A2780 cells. The Dox fluorescence level after sonication was measured in the tumor, heart, kidneys, liver and spleen and the results were compared to the case when the tumors were not sonicated. The objective of the study was to examine localized drug release controlled by US compared to the non-localized release due to the natural degradation of micelles. It was observed that micellar encapsulated Dox preferentially accumulated in the tumor, and that the tumor uptake was increased by exposure to US. Some fluorescence was detected in the liver but none in the heart. This is in contrast to what was observed when free Dox was used. Upon injection, Dox was found in the liver, spleen and all other organs of the peritoneal cavity. When insonated after injection, free Dox could be found in the tumors, kidneys, and also in the heart. These results confirmed that the cardiac toxicity of Dox can be greatly decreased when using micellar Dox instead of free Dox, and that focused US decreases the drug uptake by healthy tissues, while increasing the drug uptake locally.

Gao and co-workers [110] used unstabilized Pluronic ${ }^{\circledR}$ P105 and stabilized mixed micelles PEG- $b$-PLA (PEG2000diacylphospholipid, and PEG-co-poly(-benzyl-L-aspartate) micelles) to encapsulate Dox and study the intracellular uptake by an ovarian carcinoma A2780 tumor developed in $n u / n u$ mice. The exposure to 1- and 3-MHz HFUS 
lead to an increase in the intracellular concentration of drug in tumor cells. The combination of US and micellar Dox decreased the tumor growth rate and increased the mice survival rates.

The synergism between the use of encapsulated drugs and US was also revealed in a recent study by Hasanzadeh et al. [111] using
Micelles are a convenient carrier for hydrophobic drugs, particularly when the micelles are stabilized so they adequately retain their payload when diluted during infusion into blood or when injected directly into tissue. The Pluronic family of block copolymers has been used in micellar DDS, as well as some specialty synthesized

Table 1. Polymeric micelles drug delivery systems that have been clinically approved or are under clinical trials [112-114].

\begin{tabular}{|c|c|c|c|c|}
\hline Polymer & Drug & Therapeutic indications & $\begin{array}{l}\text { Name (clinical trial phase) or commercial } \\
\text { name (approval date) }\end{array}$ & Notes \\
\hline NA* & TLC388 & Liver, renal & Lipotecan (Phase 1/2) & \\
\hline PEG-P(Glu) & Oxaliplatin & Solid tumors, lymphoma & NC-4016 (Phase 1) & \\
\hline PEG-PLA & Cisplatin & Pancreas cancer & NC-6004 (Phase 2/3) & \\
\hline PEG-PAA & Paclitaxel & Gastric, breast cancer & NK-105 (Phase 2/3) & \\
\hline PEG-PAA & Dox & Solid tumors & NK-911 (Phase 1) & \\
\hline PEG-PPO & Dox & Advanced adenocarcinoma & SP1049C (Phase 2/3) & $\begin{array}{l}\text { Contains Pluronic as } \\
\text { ligand }\end{array}$ \\
\hline
\end{tabular}

NIPAM, N-isopropyl acrylamide ; PAA, poly(acrylic acid); PEG, polyethylene glycol; P(Asp), poly(aspartate); PGA, polyglycolic acid; P(Glu), poly(glutamic acid); PLA, poly-(Llactide); VP, vinylpyrrolidone; PPO, polypropyleneoxide.

block copolymers.

*NA - not available, property of the company.

Ultrasound at low to moderate frequencies and relatively low intensities can by used to release the payload of the micelles. This is useful in drug delivery as the US can be focused on a tumor (or other tissue) and produce localized release. Accumulation of the micelles at the targeted site also increases the local release. Accumulation can be produced passively by the EPR effect observed in some tumors, and by attaching binding ligands to the micelles. The interaction between US and micelles appears to be related to acoustic cavitation, and may be produced by fluid movement or shock waves perturbing the structure of the micelles and releasing some drug. Cells may also be perturbed by US, leading to the increased uptake of drugs or micelles via holes in cell membranes or enhanced endocytosis. Efficacy in treating tumors in vivo has been observed using doxorubicin and other drugs, but this technique has not yet been applied in human clinical trials.

There are yet some unfinished research studies that should be done before translation to clinical trials. Perhaps first, and sometimes neglected in lab research, is the fate of the block copolymers forming the micelles. Research needs to be done to show that they are safely eliminated from the body and determine the rate of clearance. Another area needful of research is accumulation of drugloaded micelles in non-targeted tissues, such as the liver, spleen and glomerulus of the kidneys. More research should also be done in the physics of adequately delivering and focusing low and mid frequency US. While high frequency US can be focused well, it cannot penetrate tissues deeply, so it may not be available to treat deep tumors.

Despite these unanswered questions, we feel that the future of controlled delivery using US and micellar carriers is promising. All 
avenues that may reduce the mortality and morbidity of cancer and the noxious side effects of conventional chemotherapy should be pursued.

\section{ABBREVIATIONS}

$\mathrm{CMC}$

$\mathrm{CW}$

$=\quad$ critical micellar concentration

DDS

$=$ continuous wave (US)

EPR

$=\quad$ drug delivery systems

Dox

$=\quad$ enhanced permeation and retention

$=$ doxorubicin 
Drug Delivery Systems Based on Polymeric Micelles and Ultrasound: A Review Current Pharmaceutical Design, 2016, Vol. 22, No. 00

\begin{tabular}{|c|c|c|}
\hline HFUS & $=$ & high frequency ultrasound \\
\hline HIFU & $=$ & high-intensity focused ultrasound \\
\hline LFUS & $=$ & low frequency ultrasound \\
\hline MePEG- $b$-PDLLA & $=$ & methoxyPEG-block-poly(D,L-lactide) \\
\hline MI & $=$ & mechanical index \\
\hline PBS & $=$ & phosphate buffered saline \\
\hline PEG & $=$ & polyethylene glycol \\
\hline \multicolumn{3}{|l|}{ PEG- $b$ - } \\
\hline p(HPMAm-Lac(n)) & $=$ & $\begin{array}{l}\text { PEG- } b \text {-poly[N-(2-hydroxypropyl) } \\
\text { methacrylamide-lactate }]\end{array}$ \\
\hline PEO & $=$ & polyethylene oxide \\
\hline PLA- $b$-PEG & $=$ & $\begin{array}{l}\text { block copolymer of poly-lactic acid and } \\
\text { polyethylene glycol }\end{array}$ \\
\hline $\mathrm{PMEO}_{2} \mathrm{MA}$ & $=$ & $\begin{array}{l}\text { poly(2-(2-methoxyethoxy)ethyl } \\
\text { methacrylate) }\end{array}$ \\
\hline pNNDEA & $=$ & poly(N,N-diethyl acrylamide) \\
\hline PPO & $=$ & polypropylene oxide \\
\hline THPMA & $=$ & 2-tetrahydropyranyl methacrylate \\
\hline US & $=$ & ultrasound \\
\hline
\end{tabular}

\section{CONFLICT OF INTEREST}

The authors report no conflict of interest regarding the publication of this paper. The authors acknowledge the financial support of the Faculty Research Grant Type 1, from American University of Sharjah (to G.A. Husseini).

\section{ACKNOWLEDGEMENTS}

Declared none.

\section{REFERENCES}

[1] Bertram JS. The molecular biology of cancer. Mol Aspects Med 2000; 21: 167-223.

[2] Wang H-Q, Jing G-J, Zheng C. Biology-constrained gene expression discretization for cancer classification. Neurocomputing 2014; 145 : 30-36.

[3] Muller PAJ, Vousden KH. p53 mutations in cancer. Nat Cell Biol 2013; 15: 2-8

[4] Jemal A, Bray F, Center MM, et al. Global cancer statistics. CA Cancer J Clin 2011; 61: 69-90.

[5] Parkin DM, Bray F, Ferlay J, et al. Global cancer statistics, 2002. CA Cancer J Clin 2005; 55: 74-108.

[6] Husseini GA, Pitt WG. Micelles and nanoparticles for ultrasonic drug and gene delivery. Adv Drug Deliv Rev 2008; 60: 1137-52.

[7] Lim EK, Jang E, Lee K, et al. Delivery of cancer therapeutics using nanotechnology. Pharmaceutics 2013; 5: 294-317.

[8] Mozafari MR, Pardakhty A, Azarmi S, et al. Role of nanocarrier systems in cancer nanotherapy. J Liposome Res 2009; 19: 310-21.

[9] Torchilin V. Multifunctional and stimuli-sensitive pharmaceutical nanocarriers. Eur J Pharm Biopharm 2009; 71: 431-44.

[10] Torchilin VP. Multifunctional nanocarriers. Adv Drug Deliv Rev 2006; 58: 1532-55.

[11] Gianella A, Read JC, Cormode DP, et al. Multifunctional Nanoparticles for Target-Specific Imaging and Therapy. In: Prud'homme S, Svenson RK, Eds. Multifunctional Nanoparticles for Drug Delivery Applications. New York: Springer 2012; pp. 155-71.

[12] Ahmed SE, Martins AM, Husseini GA. The use of ultrasound to release chemotherapeutic drugs from micelles and liposomes. J Drug Target 2015; 23: 16-42.

[13] Moussa HG, Martins AM, Husseini GA. Review on triggered liposomal drug delivery with a focus on ultrasound. Curr Cancer Drug Targets 2015; 15: 282-313.

[14] Steichen SD, Caldorera-Moore M, Peppas NA. A review of current nanoparticle and targeting moieties for the delivery of cancer therapeutics. Eur J Pharm Sci 2013; 48: 416-27.

[15] Torchilin VP. Micellar nanocarriers: pharmaceutical perspectives. Pharm Res 2007; 24: 1-16.
[16] Blanco E, Kessinger CW, Sumer BD, et al. Multifunctional micellar nanomedicine for cancer therapy. Exp Biol Med (Maywood) 2009; 234: 123-31.

[17] Cheng Y, Morshed RA, Auffinger B, et al. Multifunctional nanoparticles for brain tumor imaging and therapy. Adv Drug Deliv Rev 2014; 66: 42-57.

[18] Gandhi H, Patel VB, Mistry N, et al. Doxorubicin mediated cardiotoxicity in rats: Protective role of felodipine on cardiac indices. Environ Toxicol Pharmacol 2013; 36: 787-95.

[19] Peng F, Su Y, Ji X, et al. Doxorubicin-loaded silicon nanowires for the treatment of drug-resistant cancer cells. Biomaterials 2014; 35: 5188-95.

[20] Chlebowski RT. Adriamycin (doxorubicin) cardiotoxicity: a review. West J Med 1979; 131: 364-8.

[21] Forssen EA, Tokes ZA. Use of anionic liposomes for the reduction of chronic doxorubicin-induced cardiotoxicity. Proc Natl Acad Sci U S A 1981; 78: 1873-7.

[22] Gaucher G, Dufresne MH, Sant VP, et al. Block copolymer micelles: preparation, characterization and application in drug delivery. J Control Release 2005; 109: 169-88.

[23] Hartley GS. Aqueous solutions of paraffin-chain salts; a study in micelle formation. Paris: Hermann \& Cie 1936.

[24] Hill JP, Shrestha LK, Ishihara S, et al. Self-assembly: from amphiphiles to chromophores and beyond. Molecules 2014; 19: 8589609.

[25] Oerlemans C, Bult W, Bos M, et al. Polymeric micelles in anticancer therapy: targeting, imaging and triggered release. Pharm Res 2010; 27: 2569-89.

[26] Jones M, Leroux J. Polymeric micelles - a new generation of colloidal drug carriers. Eur J Pharm Biopharm 1999; 48: 101-11.

[27] Alexandridis P, Lindman B. Amphiphilic Block Copolymers. SelfAssembly and Applications. Amsterdam: Elsevier 2000.

[28] Adams ML, Lavasanifar A, Kwon GS. Amphiphilic block copolymers for drug delivery. J Pharm Sci 2003; 92: 1343-55.

[29] Husseini GA, Pitt WG. The use of ultrasound and micelles in cancer treatment. J Nanosci Nanotechnol 2008; 8: 2205-15.

[30] Husseini GA, Pitt WG, Martins AM. Ultrasonically triggered drug delivery: Breaking the barrier. Colloids Surf B 2014; 123C: 36486.

[31] Attwood D, Booth C, Yeates SG, et al. Block copolymers for drug solubilisation: relative hydrophobicities of polyether and polyester micelle-core-forming blocks. Int J Pharm 2007; 345: 35-41.

[32] Allen C, Maysinger D, Eisenberg A. Nano-engineering block copolymer aggregates for drug delivery. Colloids Surf B 1999; 16 : 327.

[33] Croy SR, Kwon GS. Polymeric micelles for drug delivery. Curr Pharm Des 2006; 12: 4669-84.

[34] Husseini GA, Pitt WG. Ultrasonic-activated micellar drug delivery for cancer treatment. J Pharm Sci 2009; 98: 795-811.

[35] Alakhov V, Moskaleva E, Batrakova EV, et al. Hypersensitization of multidrug resistant human ovarian carcinoma cells by pluronic P85 block copolymer. Bioconjug Chem 1996; 7: 209-16.

[36] Batrakova EV, Dorodnych TY, Klinskii EY, et al. Anthracycline antibiotics non-covalently incorporated into the block copolymer micelles: in vivo evaluation of anti-cancer activity. Br J Cancer 1996; 74: $1545-52$.

[37] Venne A, Li S, Mandeville R, et al. Hypersensitizing effect of pluronic L61 on cytotoxic activity, transport, and subcellular distribution of doxorubicin in multiple drug-resistant cells. Cancer Res 1996; 56: 3626-9.

[38] Pruitt JD, Husseini G, Rapoport N, et al. Stabilization of Pluronic P105 micelles with an interpenetrating network of N,Ndiethylacrylamide. Macromolecules 2000; 33: 9306-9.

[39] Husseini GA, Christensen DA, Rapoport NY, et al. Ultrasonic release of doxorubicin from Pluronic P105 micelles stabilized with an interpenetrating network of $\mathrm{N}, \mathrm{N}$-diethylacrylamide. J Control Release 2002; 83: 303-5.

[40] Zeng Y, Pitt, WG Poly(ethylene oxide)-bpoly(nisopropylacrylamide) nanoparticles with crosslinked cores as drug carriers. J Biomat Sci Polym 2005; 16: 371-80.

[41] Xuan J, Pelletier M, Xia H, et al. Ultrasound-induced disruption of amphiphilic block copolymer micelles. Macromol Chem Phys 2011; 212: 498-506.

[42] Zhang $\mathrm{H}$, Xia $\mathrm{H}$, Wang $\mathrm{J}$, et al. High intensity focused ultrasoundresponsive release behavior of PLA-b-PEG copolymer micelles. J Control Release 2009; 139: 31-9. 

induced by micellar-delivered doxorubicin and ultrasound: comet assay study. Cancer Lett 2000; 154: 211-6.

[44] Pitt WG, Husseini GA, Staples BJ. Ultrasonic drug delivery--a general review. Expert Opin Drug Deliv 2004; 1: 37-56.

[45] Mitragotri S. Healing sound: the use of ultrasound in drug delivery and other therapeutic applications. Nat Rev Drug Discov 2005; 4: 255-60.

[46] Schroeder A, Kost J, Barenholz Y. Ultrasound, liposomes, and drug delivery: principles for using ultrasound to control the release of drugs from liposomes. Chem Phys Lipids 2009; 162: 1-16.

[47] Ahmadi F, McLoughlin IV, Chauhan S, et al. Bio-effects and safety of low-intensity, low-frequency ultrasonic exposure. Prog Biophys Mol Biol 2012; 108: 119-38.

[48] Dalecki D. Mechanical bioeffects of ultrasound. Annu Rev Biomed Eng 2004; 6: 229-48.

[49] Jenne JW, Preusser T, Gunther M. High-intensity focused ultrasound: principles, therapy guidance, simulations and applications. Z Med Phys 2012; 22: 311-22.

[50] Husseini GA, Stevenson-Abouelnasr D, Pitt WG, et al. Kinetics and thermodynamics of acoustic release of doxorubicin from nonstabilized polymeric micelles. Colloids Surf A 2010; 359: 18-24.

[51] Alexandrov AV. Ultrasound enhancement of fibrinolysis. Stroke 2009; 40: S107-10.

[52] Draper DO, Castel JC, Castel D. Rate of temperature increase in human muscle during $1 \mathrm{MHz}$ and $3 \mathrm{MHz}$ continuous ultrasound. J Orthop Sports Phys Ther 1995; 22: 142-50.

[53] de Smet $\mathrm{M}$, Langereis $\mathrm{S}$, van den Bosch $\mathrm{S}$, et al. Temperaturesensitive liposomes for doxorubicin delivery under MRI guidance.

J Control Release 2010; 143: 120-7.

[54] Gasselhuber A, Dreher MR, Partanen A, et al. Targeted drug delivery by high intensity focused ultrasound mediated hyperthermia combined with temperature-sensitive liposomes: computational modelling and preliminary in vivo validation. Int $\mathrm{J}$ Hyperthermia 2012; 28: 337-48.

[55] Park SM, Kim MS, Park S-J, et al. Novel temperature-triggered liposome with high stability: Formulation, in vitro evaluation, and in vivo study combined with high-intensity focused ultrasound (HIFU). J Control Release 2013; 170: 373-9.

[56] Arvanitis CD, Bazan-Peregrino M, Rifai B, et al. CavitationEnhanced Extravasation for Drug Delivery. Ultrasound Med Biol 2011; 37: 1838-52.

[57] Husseini GA, Diaz de la Rosa MA, Richardson ES, et al. The role of cavitation in acoustically activated drug delivery. J Control Release 2005; 107: 253-61.

[58] Brennen CE. Cavitation and Bubble Dynamics. New York: Oxford University Press 1995; pp. 282

[59] Stevenson-Abouelnasr D, Husseini GA, Pitt WG. Further investigation of the mechanism of Doxorubicin release from P105 micelles using kinetic models. Colloids Surf, B 2007; 55: 59-66.

[60] Husseini GA, Velluto D, Kherbeck L, et al. Investigating the acoustic release of doxorubicin from targeted micelles. Colloids Surf B 2013; 101: 153-5.

[61] Maeda H. The enhanced permeability and retention (EPR) effect in tumor vasculature: the key role of tumor-selective macromolecular drug targeting. Adv Enzyme Regul 2001; 41: 189-207.

[62] Maeda H, Wu J, Sawa T, et al. Tumor vascular permeability and the EPR effect in macromolecular therapeutics: a review. J Control Release 2000; 65: 271-84.

[63] Webster DM, Sundaram P, Byrne ME. Injectable nanomaterials for drug delivery: carriers, targeting moieties, and therapeutics. Eur J Pharm Biopharm 2013; 84: 1-20.

[64] Allen TM. Ligand-targeted therapeutics in anticancer therapy. Nat Rev Cancer 2002; 2: 750-63.

[65] Byrne JD, Betancourt T, Brannon-Peppas L. Active targeting schemes for nanoparticle systems in cancer therapeutics. Adv Drug Deliv Rev 2008; 60: 1615-26.

[66] Torchilin VP. Targeted pharmaceutical nanocarriers for cancer therapy and imaging. AAPS J 2007; 9: E128-47.

[67] Hilgenbrink AR, Low PS. Folate receptor-mediated drug targeting: from therapeutics to diagnostics. J Pharm Sci 2005; 94: 2135-46.

[68] Leamon CP, Reddy JA. Folate-targeted chemotherapy. Adv Drug Deliv Rev 2004; 56: 1127-41.

[69] Sudimack J, Lee RJ. Targeted drug delivery via the folate receptor. Adv Drug Deliv Rev 2000; 41: 147-62.

\section{Current Pharmaceutical Design, 2016, Vol. 22, No. 00}

[70] Zhao X, Li H, Lee RJ. Targeted drug delivery via folate receptors. Expert Opin Drug Deliv 2008; 5: 309-19.

[71] Shen Z, Li Y, Kohama K, et al. Improved drug targeting of cancer cells by utilizing actively targetable folic acid-conjugated albumin nanospheres. Pharmacol Res 2011; 63: 51-8.

[72] Yu MK, Park J, Jon S. Targeting strategies for multifunctional nanoparticles in cancer imaging and therapy. Theranostics 2012; 2 : $3-44$.

[73] Ai J, Xu Y, Li D, et al. Folic acid as delivery vehicles: targeting folate conjugated fluorescent nanoparticles to tumors imaging. Talanta 2012; 101: 32-7.

[74] Yu B, Tai HC, Xue W, et al. Receptor-targeted nanocarriers for therapeutic delivery to cancer. Mol Membr Biol 2010; 27: 286-98.

[75] Rapoport N. Physical stimuli-responsive polymeric micelles for anticancer drug delivery. Prog Polym Sci 2007; 32: 962-90.

[76] Husseini GA, Myrup GD, Pitt WG, et al. Factors affecting acoustically triggered release of drugs from polymeric micelles. J Control Release 2000; 69: 43-52.

[77] Diaz de la Rosa MA, Husseini GA, Pitt WG. Mathematical modeling of microbubble cavitation at $70 \mathrm{kHz}$ and the importance of the subharmonic in drug delivery from micelles. Ultrasonics 2013; 53: 97-110.

[78] Husseini GA, Kherbeck L, Pitt WG, et al. Kinetics of Ultrasonic Drug Delivery from Targeted Micelles. J Nanosci Nanotechnol 2015; 15: 2099-104.

[79] Diaz de la Rosa MA, Husseini GA, Pitt WG. Comparing microbubble cavitation at $500 \mathrm{kHz}$ and $70 \mathrm{kHz}$ related to micellar drug delivery using ultrasound. Ultrasonics 2013; 53: 377-86.

[80] Stringham SB, Viskovska MA, Richardson ES, et al. Over-pressure suppresses ultrasonic-induced drug uptake. Ultrasound Med Biol 2009; 35: 409-15.

[81] Husseini GA, Rapoport NY, Christensen DA, et al. Kinetics of ultrasonic release of doxorubicin from pluronic P105 micelles. Colloids Surf B 2002; 24: 253.

[82] Husseini GA, Abdel-Jabbar NM, Mjalli FS, et al. Optimizing the use of ultrasound to deliver chemotherapeutic agents to cancer cells from polymeric micelles. J Franklin Inst 2011; 348: 1276-84.

[83] Husseini GA, Diaz de la Rosa MA, Gabuji T, et al. Release of doxorubicin from unstabilized and stabilized micelles under the action of ultrasound. J Nanosci Nanotechnol 2007; 7: 1028-33.

[84] Ugarenko M, Chan CK, Nudelman A, et al. Development of pluronic micelle-encapsulated doxorubicin and formaldehydereleasing prodrugs for localized anticancer chemotherapy. Oncol Res 2009; 17 : 283-99.

[85] Xuan J, Boissiere O, Zhao Y, et al. Ultrasound-responsive block copolymer micelles based on a new amplification mechanism. Langmuir 2012; 28: 16463-8.

[86] Wang J, Pelletier $\mathrm{M}$, Zhang $\mathrm{H}$, et al. High-frequency ultrasoundresponsive block copolymer micelle. Langmuir 2009; 25: 13201-5.

[87] Deckers R, Paradissis A, Oerlemans C, et al. New insights into the HIFU-triggered release from polymeric micelles. Langmuir 2013; 29: 9483-90.

[88] Abdel-Hafez M, Husseini G. Predicting the Release of Chemotherapeutics From the Core of Polymeric Micelles Using Ultrasound. IEEE Trans Nanobioscience 2015.

[89] Munshi N, Rapoport N, Pitt WG. Ultrasonic activated drug delivery from Pluronic P-105 micelles. Cancer Lett 1997; 118: 13-9.

[90] Marin A, Muniruzzaman M, Rapoport N. Acoustic activation of drug delivery from polymeric micelles: effect of pulsed ultrasound. J Control Release 2001; 71: 239-49.

[91] Marin A, Sun H, Husseini GA, et al. Drug delivery in pluronic micelles: effect of high-frequency ultrasound on drug release from micelles and intracellular uptake. J Control Release 2002; 84: 3947.

[92] Howard B, Gao, A., Lee S.-W., et al. Ultrasound-enhanced chemotherapy of drug-resistant breast cancer tumors by micellarencapsulated paclitaxel. Am J Drug Deliv 2006; 4: 97-104.

[93] Wan CP, Jackson JK, Pirmoradi FN, et al. Increased accumulation and retention of micellar paclitaxel in drug-sensitive and Pglycoprotein-expressing cell lines following ultrasound exposure. Ultrasound Med Biol 2012; 38: 736-44.

[94] Tachibana K, Uchida T, Tamura K, et al. Enhanced cytotoxic effect of Ara-C by low intensity ultrasound to HL-60 cells. Cancer Lett 2000; 149: 189-94. 
[95] Yamashita N, Tachibana K, Ogawa K, et al. Scanning electron microscopic evaluation of the skin surface after ultrasound exposure. Anat Rec 1997; 247: 455-61.

[96] Tachibana K, Uchida T, Ogawa K, et al. Induction of cellmembrane porosity by ultrasound. Lancet 1999; 353: 1409.

[97] Schlicher RK, Hutcheson JD, Radhakrishna H, et al. Changes in cell morphology due to plasma membrane wounding by acoustic cavitation. Ultrasound Med Biol 2010; 36: 677-92.

[98] Schlicher RK, Radhakrishna H, Tolentino TP, et al. Mechanism of intracellular delivery by acoustic cavitation. Ultrasound Med Biol 2006; 32: 915-24.

[99] Zhou Y, Kumon RE, Cui J, et al. The size of sonoporation pores on the cell membrane. Ultrasound Med Biol 2009; 35: 1756-60.

[100] Hauser J, Ellisman M, Steinau HU, et al. Ultrasound enhanced endocytotic activity of human fibroblasts. Ultrasound Med Biol 2009; 35: 2084-92.

[101] Meijering BD, Juffermans LJ, van Wamel A, et al. Ultrasound and microbubble-targeted delivery of macromolecules is regulated by induction of endocytosis and pore formation. Circ Res 2009; 104: 679-87.

[102] Muniruzzaman MD, Marin A, Luo Y, et al. Intracellular uptake of pluronic copolymer: effects of the aggregation state. Colloids Surf B 2002; 25: 233-41.

[103] Rapoport N. Combined cancer therapy by micellar-encapsulated drug and ultrasound. Int J Pharm 2004; 277: 155-62.

[104] Sheikov N, McDannold N, Jolesz F, et al. Brain arterioles show more active vesicular transport of blood-borne tracer molecules than capillaries and venules after focused ultrasound-evoked opening of the blood-brain barrier. Ultrasound Med Biol 2006; 32: 1399-409.

[105] Lentacker I, De Cock I, Deckers R, et al. Understanding ultrasound induced sonoporation: Definitions and underlying mechanisms. Adv Drug Deliv Rev 2014; 72: 49-64.

[106] Nelson JL, Ultrasonically Enhanced Drug Delivery of Doxorubicin in vivo from Stabilized Pluronic Micelle Carriers, M.Sc. Thesis. Provo, UT: Brigham Young University 2002.

[107] Staples BJ, Roeder BL, Husseini GA, et al. Role of frequency and mechanical index in ultrasonic-enhanced chemotherapy in rats. Cancer Chemother Pharmacol 2009; 64: 593-600.

[108] Staples BJ, Pitt WG, Roeder BL, et al. Distribution of doxorubicin in rats undergoing ultrasonic drug delivery. J Pharm Sci 2010; 99: 3122-31.

[109] Rapoport NY, Christensen DA, Fain HD, et al. Ultrasoundtriggered drug targeting of tumors in vitro and in vivo. Ultrasonics 2004; 42: 943-50.

[110] Gao ZG, Fain HD, Rapoport N. Controlled and targeted tumor chemotherapy by micellar-encapsulated drug and ultrasound. J Control Release 2005; 102: 203-22.

[111] Hasanzadeh H, Mokhtari-Dizaji M, Bathaie SZ, et al. Effect of local dual frequency sonication on drug distribution from polymeric nanomicelles. Ultrason Sonochem 2011; 18: 1165-71.

[112] Wicki A, Witzigmann D, Balasubramanian V, et al. Nanomedicine in cancer therapy: Challenges, opportunities, and clinical applications. J Control Release 2015; 200: 138-57.

[113] Cho K, Wang X, Nie S, et al. Therapeutic nanoparticles for drug delivery in cancer. Clin Cancer Res 2008; 14: 1310-6.

[114] Slowing II, Vivero-Escoto JL, Wu C-W, et al. Mesoporous silica nanoparticles as controlled release drug delivery and gene transfection carriers. Adv Drug Deliv Rev 2008; 60: 1278-88. 